\begin{tabular}{llllllllll}
\multicolumn{1}{ll}{ Absreact PMO-9 Table 1} & & & & & \\
\hline
\end{tabular}

Key: Pt - patients, Inf - infusions, MAb - monoclonal antibody, Induct - Induction, Maint - maintenance, IV - intravenous, PO - oral, NC - not collected

anecdotally improved. Further data from other centres are required to prove significance.

\section{PMO-10 SURVEY ON THE USE OF ARTIFICIAL INTELLIGENCE IN IBD PATIENTS IN THE USA AND UK}

${ }^{1}$ Gaurav Nigam*, ${ }^{2}$ Rajan Patel, ${ }^{3}$ Raj G Jatale, ${ }^{4}$ Brian Bressler, ${ }^{5}$ Bu Hayee, ${ }^{6}$ Marietta lacucci, ${ }^{7}$ Francis A Farraye, ${ }^{1}$ Jimmy Limdi. ${ }^{1}$ The Pennine Acute Hospitals NHS Trust, Manchester, UK; ${ }^{2}$ Royal Free London NHS Trust, London, UK; ${ }^{3}$ P D Hinduja Hospital, Mumbai, India; ${ }^{4}$ University of British Columbia, Vancouver, Canada; ${ }^{5}$ King's College Hospital NHS Foundation Trust, London, UK; ${ }^{6}$ University Hospitals Birmingham NHS Foundation Trust, Birmingham, UK; ${ }^{7}$ Inflammatory Bowel Disease Center, Mayo Clinic, Jacksonville, USA

\subsection{6/gutjnl-2021-BSG. 149}

Introduction Artificial intelligence (AI) is making rapid in-roads in various aspects of gastroenterology (GI). Early studies have shown potential for the use of AI in the diagnosis and management of inflammatory bowel disease (IBD). Our aim was to explore the current understanding of clinicians for the role of $\mathrm{AI}$ in GI and IBD in particular.

Methods A 15-question survey was developed in consultation amongst the authors and distributed to members of the American College of Gastroenterology (ACG) \& British Society of Gastroenterology (BSG) in May 2020. The questionnaire was approved by the ACG Research Committee and the BSG IBD Committee for the USA and the UK, respectively. Data was analysed using R software Version 3.5.2.

Results A total of 249 members (USA-175, UK-74) responded. IBD surveillance colonoscopies were being performed by $84.7 \%$. A total of $171(68.7 \%)$ respondents were aware of the potential use of AI in GI. Specifically, 140 (81.9\%) were aware of current use of AI for colonic polyp detection, 82 (47.9\%) for Barrett's surveillance, 72 (42.1\%) for capsule endoscopy, $41(24 \%)$ in early gastric cancer detection and 7 (4.1\%) for IBD.

Furthermore, $86.5 \%$ thought that AI could potentially improve IBD care in the future. The 3 most unmet needs in surveillance colonoscopy in patients with IBD were appropriate surveillance intervals $(58.6 \%)$; accurate histopathology and dysplasia detection (57.4\%); and yield from different biopsy protocols (51.4\%). Suggested areas for use of $\mathrm{AI}$ in IBD were real time assessment and endoscopic scoring (73.1\%), earlier detection of colorectal cancer (70.2\%), facilitating 'personalised' care (50.9\%) and

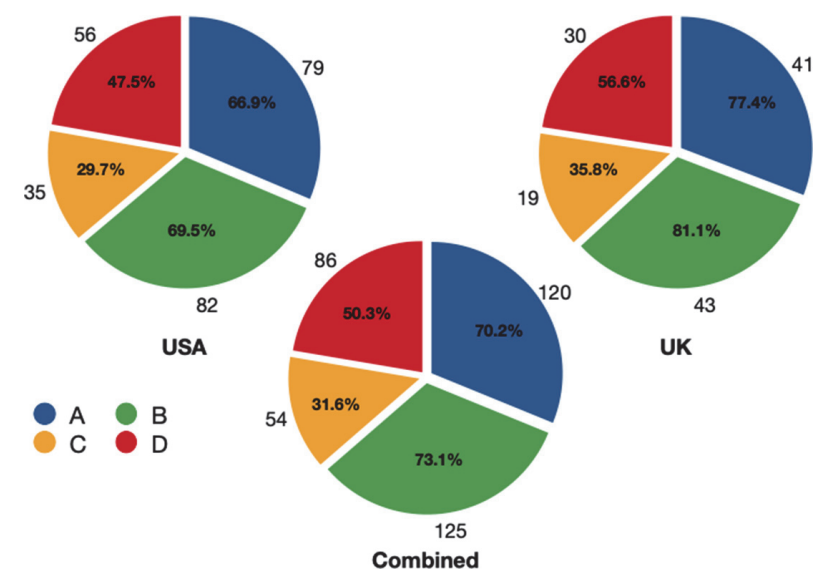

Abstract PM0-10 Figure 1 Suggested areas for use Al in IBD

distinguishing Crohn's disease from ulcerative colitis at index colonoscopy (31.6\%).

Respondents projected that AI would be available in clinical practice for IBD soon; $13.4 \%$ in $<1$ year; $34.5 \%<2$ years and $52.1 \%<5$ years. The potential perceived barriers for use of AI in gastroenterology were cost (66.7\%), uncertainty about technology (61.4\%) and access to AI courses (47.3\%). Respondents had concerns regarding patient safety with use of AI $(26.3 \%)$ and concerns regarding patient confidentiality (39.8\%).

Conclusions There is a high level of awareness for AI in polyp detection but significantly less in IBD. Respondents felt that AI could improve endoscopic assessment in IBD, dysplasia surveillance and aid personalised care. Cost, unfamiliarity with AI technology and access to AI courses were perceived as likely barriers.

\section{PMO-11 A SIMPLE TOOL IMPROVES SCREENING FOR PSYCHOLOGICAL DISTRESS IN OUTPATIENTS WITH INFLAMMATORY BOWEL DISEASE}

1J Kennedy, 'Jessica Toombs*, ${ }^{2} S$ Hepburn, ${ }^{2}$ A Wroe, ${ }^{1} \mathrm{~A}$ De Silva. ${ }^{1}$ Department of Gastroenterology, Royal Berkshire NHS Foundation Trust, Reading, UK; ${ }^{2}$ Clinical Health Psychology Service, Berkshire Healthcare NHS Foundation Trust, Reading, UK

10.1136/gutjnl-2021-BSG. 150 
Introduction Psychological distress is highly prevalent in patients living with inflammatory bowel disease (IBD). Identifying and managing psychological distress can improve IBD symptoms and quality of life and reduce interaction with secondary care ${ }^{1}$. IBD standards have long emphasised the importance of psychological support, particularly at the time of diagnosis, in patients experiencing pain or fatigue or in patients being considered for surgery ${ }^{2}$.

This project aimed to improve screening for psychological distress in outpatients living with IBD at a large district general hospital, whilst also increasing the number of patients signposted or referred to support services if indicated.

Methods Outpatient letters from 167 consecutive IBD consultations between October 2019 and November 2020 were retrospectively reviewed to see if psychological distress was discussed and, if indicated, whether patients were referred for support. A three question screening tool was then developed to identify patients suffering from psychological distress, with a clear referral pathway if required to either the clinical health IBD psychology service or the local Improving Access to Psychological Therapies service. The tool was promoted to healthcare professionals seeing patients with IBD. 66 postintervention clinic letters were then reviewed. The second cycle involved saving the tool as a template to the patient record prior to an appointment to act as a prompt for screening.

Results Psychological distress was discussed in significantly more consultations post-intervention than pre-intervention $(32.8 \%$ vs $15.6 \% \mathrm{p}=0.004)$. The bespoke screening tool was used in $23 \%$ of consultations post-intervention. Of those screened $21.4 \%$ reported psychological distress. The intervention increased the frequency of signposting and referral of patients with psychological distress from $35.7 \%$ to $87.5 \%$ $(\mathrm{p}=0.019)$. There was no significant difference in rate of screening between cycle 1 and 2 (44.4\% vs $41.4 \% \mathrm{p}=0.766)$; demonstrating the change was sustained.
Conclusions Clinician education and a simple outpatient screening tool can significantly improve screening for psychological distress and subsequently identify and refer on those patients with the greatest need. We intend to increase awareness of our screening tool and our next intervention involves digitising the tool; enabling patients to complete it prior to their clinic appointment. We will also evaluate the impact of our service on health-related quality of life in our patient population.

\section{REFERENCE}

1. McGeer R, Ascott A, Smith M, et al. OUT-034 IBD psychological support pilot reduces IBD symptoms and improves psychological wellbeing. Gut 2018;67:A224A226. https://ibduk.org/ibd-standards accessed 16/12/2020

\section{PM0-12 REAL-WORLD OUTCOMES AFTER VEDOLIZUMAB DISCONTINUATION IN A TERTIARY IBD COHORT}

${ }^{1}$ Gregory Sebepos-Rogers*, ${ }^{1}$ Konstantinos Fragkos, ${ }^{1}$ Hajeena Saravanapavan, ${ }^{1}$ John Barragry, ${ }^{2}$ Hui Fen Koo, ${ }^{2}$ Ho Juen Ko, ${ }^{1}$ loanna Parisi, ${ }^{1}$ Edward Seward, ${ }^{1}$ Sara McCartney, ${ }^{1}$ Stuart Bloom, ${ }^{1}$ Shameer Mehta, ${ }^{1}$ Faroog Rahman. ${ }^{1}$ Dept. Gastroenterology, University College Hospital, London, UK; ${ }^{2}$ University College London Medical School, London, UK

\subsection{6/gutjnl-2021-BSG.151}

Introduction Vedolizumab. (VDZ) is an $\alpha 4 \beta 7$ integrin antagonist licensed to treat moderate to severe ulcerative colitis (UC) and Crohn's disease (CD). We provide long-term real-world data on outcomes after VDZ discontinuation.

Methods A consecutive cohort of 193 adult IBD patients initiated on VDZ between May 2015 and June 2019 was retrospectively reviewed up to June 2020. Primary outcomes were post-VDZ relapse, biologic and surgical event rates. VDZ discontinuation for remission was classified as treatment for at least 6 months with 3 months corticosteroid-free clinical remission pre-discontinuation. Statistics: Continuous variables

Abstract PM0-12 Table 1 Post vedolizumab treatment options in CD $(n=67)$ and UC $(n=59)$

\begin{tabular}{|c|c|c|c|c|c|c|c|}
\hline & Infliximab & Adalimumab & Ustekinumab & Tofacitinib & $\begin{array}{l}\text { Vedolizumab } \\
\text { re-use }\end{array}$ & Golimumab & Surgery \\
\hline $\mathrm{CD}(n=67)$ & $3(4.5 \%)$ & $4(6 \%)$ & $44(65.7 \%)$ & $1(1.5 \%)$ & $4(6 \%)$ & $0(0 \%)$ & $\begin{array}{l}26 \\
(38.8 \%)\end{array}$ \\
\hline Persistence & $2(67 \%)$ & $3(75 \%)$ & $30(68 \%)$ & $0(0 \%)$ & $2(50 \%)$ & $0(0 \%)$ & \\
\hline Surgery & $1(33 \%)$ & $2(50 \%)$ & $14(32 \%)$ & $0(0 \%)$ & $2(50 \%)$ & $0(0 \%)$ & \\
\hline \multicolumn{8}{|c|}{ Number of previous biologics } \\
\hline 0 & $0(0 \%)$ & $2(3 \%)$ & $0(0 \%)$ & $0(0 \%)$ & $0(0 \%)$ & $0(0 \%)$ & $0(0 \%)$ \\
\hline 1 & $2(3 \%)$ & $0(0 \%)$ & $15(22.4 \%)$ & $0(0 \%)$ & $2(3 \%)$ & $0(0 \%)$ & $5(7.5 \%)$ \\
\hline 2 & $1(1.5 \%)$ & $0(0 \%)$ & $26(38.8 \%)$ & $1(1.5 \%)$ & $1(1.5 \%)$ & $0(0 \%)$ & $\begin{array}{l}16 \\
(23.9 \%)\end{array}$ \\
\hline 3 & $0(0 \%)$ & $2(3 \%)$ & $3(4.5 \%)$ & $0(0 \%)$ & $1(1.5 \%)$ & $0(0 \%)$ & $5(7.5 \%)$ \\
\hline $\begin{array}{l}\chi^{2} p \text {-value } \\
\text { Disease Loca }\end{array}$ & 0.473 & $<0.0001$ & $<0.0001$ & 0.775 & 0.700 & - & 0.184 \\
\hline Ileal & $1(1.5 \%)$ & $0(0 \%)$ & $8(11.9 \%)$ & $0(0 \%)$ & $2(3 \%)$ & $0(0 \%)$ & $2(3 \%)$ \\
\hline Colonic & $2(3 \%)$ & $4(6 \%)$ & $36(53.7 \%)$ & $1(1.5 \%)$ & $2(3 \%)$ & $0(0 \%)$ & $\begin{array}{l}24 \\
(35.8 \%)\end{array}$ \\
\hline$\chi^{2}$ p-value & 0.418 & 0.361 & 0.59 & 0.655 & 0.062 & - & 0.125 \\
\hline UC $(n=59)$ & $16(27.1 \%)$ & $11(18.6 \%)$ & $5(8.5 \%)$ & $11(18.6 \%)$ & $4(6.8 \%)$ & $1(1.7 \%)$ & $\begin{array}{l}16 \\
(27.2 \%)\end{array}$ \\
\hline
\end{tabular}

